\title{
Development of a Real-Time Driver Health Detection System Using a Smart Steering Wheel
}

\author{
Jae-Cheon Lee ${ }^{1}$, Hao Liu ${ }^{2}$ \\ ${ }^{1,2}$ Dept. of Mechanical and Automotive Engineering, Keimyung University, Daegu, 42601, S. Korea \\ ljcds@kmu.ac.kr \\ liuhao@kmu.ac.kr
}

\begin{abstract}
The number of vehicle accidents due to driver drowsiness continues to increase. Therefore, prompt and effective detection for driver health during driving is crucial to improvement of traffic safety. A set of real-time health detection system built into a smart steering wheel for the driver is proposed in the paper. The driver's health condition (drowsiness) is detected by a developed algorithm by monitoring the driver's biological signals, including respiration, hand grip force, photoplethysmogram (PPG), and electrocardiogram (ECG). Meanwhile the driver's state of arrhythmia, as a common cardiac disease, can be diagnosed too. The test results indicate that the developed real-time driver health detection system can effectively monitor the state of vigilance and the cardiac state, i.e. arrhythmia, of the driver.
\end{abstract}

\section{INTRODUCTION}

The Traffic Accident Analysis Center (2012) reports that the number of traffic accidents caused by driver carelessness has gradually increased. Drowsiness and fatigue have become one of major reasons for serious traffic accidents. According to the U.S. National Highway Traffic Safety Administration (NHTSA), drowsiness during driving is responsible for at least 100,000 vehicle crashes annually, resulting in annual averages of roughly 40,000 nonfatal injuries and 1,550 fatalities (Bergasa, Nuevo, Sotelo, Barea, and Lopez, 2006). The National Sleep Foundation also reports that $60 \%$ of adult drivers have driven while feeling drowsy, and $37 \%$ have even actually fallen asleep at the wheel (Rosekind, 2006). Thus, traffic accidents due to driver drowsiness have become a serious social problem. Moreover, driver's serious disease such as heart attacks could also lead to fatal traffic accidents.

Jae-Cheon Lee et al. This is an open-access article distributed under the terms of the Creative Commons Attribution 3.0 United States License, which permits unrestricted use, distribution, and reproduction in any medium, provided the original author and source are credited.
As a result, it is significant to detect driver health efficiently and in real time during driving in order to improve of traffic safety. Researchers have developed many methods to monitor driver's state of vigilance. Once the driver is considered to be falling asleep, the equipment can wake up the driver by giving warning signals via strong seat vibration or a high pitch sound so as to prevent vehicle accidents. Eskandarian and Mortazavi (2007) monitored driver vigilance based on driving performance to estimate driver fatigue. Other methods based on monitoring eye activity with video cameras have also been developed to monitor driver vigilance.

The most reliable monitoring method is to use physiological signals such as brain wave, heart rate, pulse rate, and respiration rate signals. In this study, considering convenience of monitoring the driver as driving, respiration, hand gripping force, and electrocardiogram (ECG) signals are utilized to detect wake or sleep stages of the driver by analyzing Heart Rate (HR) and Heart Rate Variability (HRV). Driver's physiological signals were monitored via a smart steering wheel and analyzed to determine driver's health state. This is the reason why we call the developed steering wheel with acquisition and analysis of driver's physiological signals as the smart steering wheel. The paper layout is as below. The construction of the monitoring system is presented in section 2, and the detection algorithm is presented in section 3. The last section presents conclusions and future work.

\section{REAL-TIME DRIVER HEALTH DETECTION SYSTEM}

The proposed real-time health detection system applied onto the smart steering wheel is composed of three biological sensors, an interface circuit, a data acquisition board (DAQ), and a personal computer (PC). The system can monitor driver's biological signals, including respiration, grip force, PPG, and ECG signals. The layout of the entire smart steering wheel for real-time driver's health detection system is shown in Figure 1. 


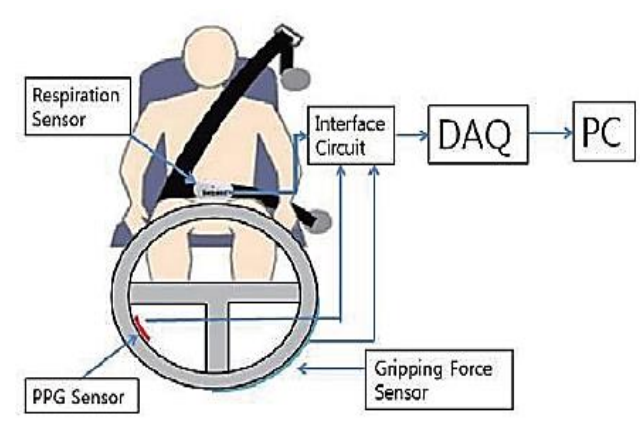

Figure 1. Layout of smart steering wheel system for real-time driver health detection

\subsection{Sensors for Monitoring Driver Health}

The smart steering wheel uses three kinds of biological sensors: pressure sensors, a PPG Sensor, and ECG detection probes. The respiration signal and grip force are obtained from pressure sensors attached to the seat belt and the steering wheel, respectively. The PPG signal is acquired from a PPG sensor, which consists of a light-emitting diode (LED) and a phototransistor (PT) attached to the steering wheel. The ECG signal is obtained by two probes attached to the steering wheel and a probe on the backrest.

The respiration signal is an important and easy-to-measure physiological signal. The pressure sensor made from a wire resistor and is bonded to the abdomen part of the safety belt to measure driver respiration, as illustrated in Figure 2. Resistance variation of the pressure sensor can be measured when the driver inhales and exhales, so the respiration signal can be detected. A correlation has recently been found between respiration and drowsiness. Therefore, based on the respiration signal, driver drowsiness can be determined.
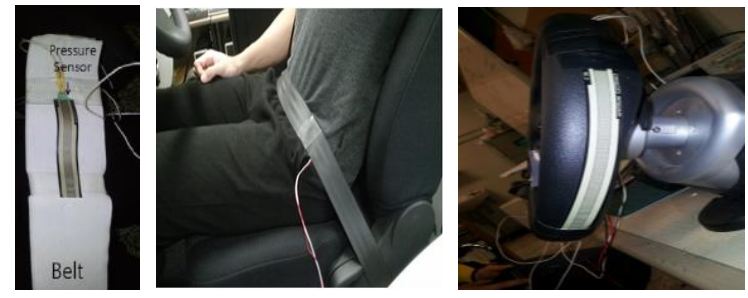

Figure 2. Pressure sensor attached to the safety belt and the smart steering wheel.

Figure 3 shows the PPG sensor, including a light-emitting diode (LED) and a phototransistor (PT), attached to the steering wheel. As the driver's hands hold the smart steering wheel, light generated by the LED is shone onto the skin, and the amount of light reflected to the PT can be measured. The measured light depends on the volume change of the blood vessels induced by the pressure pulse of the cardiac cycle. By using information obtained from the PPG sensor, the driver heart rate and heart rate variability can be obtained.

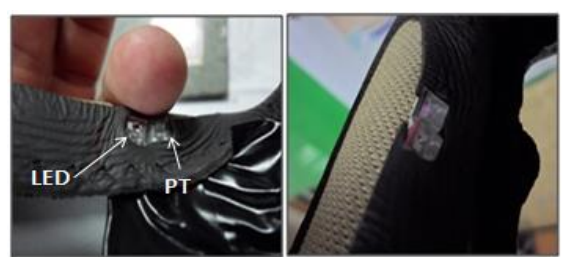

Figure 3. PPG sensor including LED and PT

Two patches of conductive fiber (electrodes) are attached to the smart steering wheel (Figure 4) to detect the tiny electrical changes on driver's skin that arise from the heart muscle's electrophysiologic pattern of depolarizing and repolarizing during each heartbeat. In order to ensure to obtain reliable ECG signal, an additional probe is installed on the driver seat backrest.
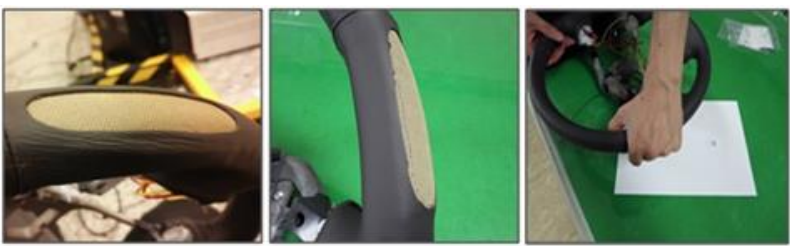

Figure 4. ECG detection probes made from conductive fiber

\subsection{Interface Circuit and Data Acquisition Board}

An interface circuit is necessary to convert signals acquired by sensors to proper analogue signals for reading by a data acquisition board. Other functions of the interface circuit are also to filter and amplify signals. For example, waveforms with frequencies between 0.15 and $4 \mathrm{~Hz}$ are main components of a human ECG signal. Therefore, the ECG signal was conditioned using a high-pass filter with a cutoff frequency of $0.15 \mathrm{~Hz}$, a low-pass filter with a cutoff frequency of $4 \mathrm{~Hz}$, and a notch filter for getting rid of noise at $50 \mathrm{~Hz}$. The filtered ECG signal is then amplified by about 20 times. The interface circuit for the ECG signal is shown in Figure 5.

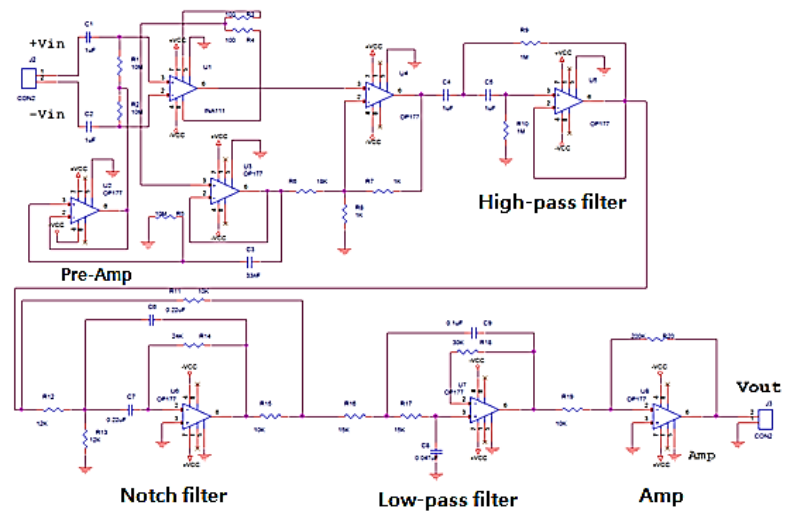

Figure 5. Interface circuit for ECG signal conditioning

A 10-bit data acquisition board and PC are constructed for hardware part and the monitoring graphic user interface 
(GUI) is developed. Figure 6 presents the signals acquired by various sensors and presented in the GUI. Due to the huge risk of measuring signals from an actual drowsy driver during driving, test signals were acquired from measurements in a laboratory instead of from real drivers in vehicles.

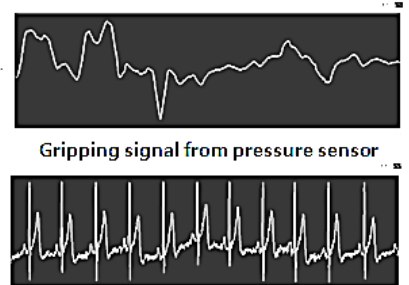

ECG signal from probes

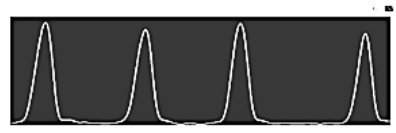

Respiration signal

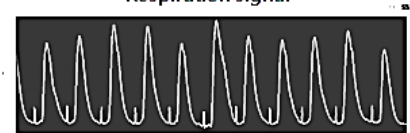

Signal from PPG sensor
Figure 6. Signal representation in GUI

\section{DEVELOPMENT OF DETECTION ALGORITHM}

Based on the previous hardware construction of the realtime driver health detection system, the next work is to develop detection algorithm of driver drowsiness (vigilance) and arrhythmia.

\subsection{Drowsiness Detection}

The peak value and moment of the acquired respiration signal are firstly determined, and then the respiration rate can be calculated from the continuous respiration signal. The peak value and respiration rates when awake and sleepy are different, so reference values can be used to judge whether or not the driver gets into drowsy. The number of drowsiness signals is counted, and if it reaches three, the driver is considered to be drowsy. The entire detection process is illustrated in Figure 7.

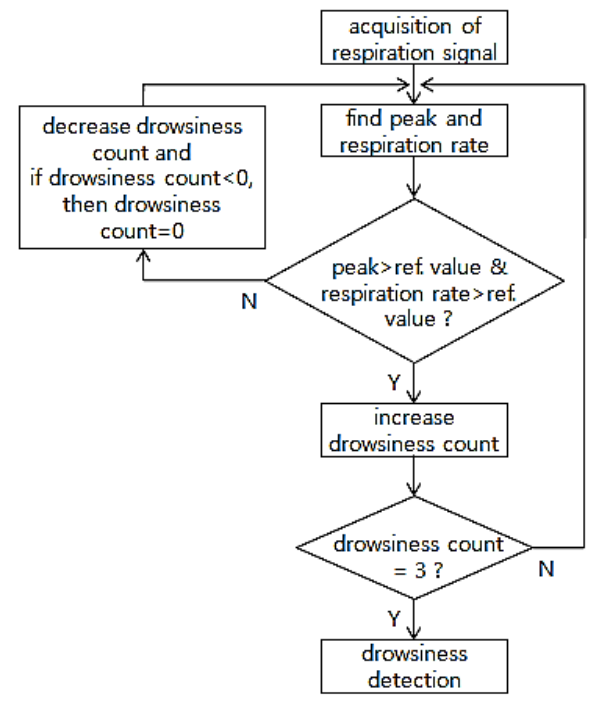

Figure 7. Drowsiness detection algorithm based on respiration signals
The PPG signal peak (shown in Figure 6) can also be found, and some statistical analysis is performed, such as the average and dispersion of the peak-peak interval. Similarly, these two statistical quantities are compared with reference values to detect driver drowsiness. The flow chart of the algorithm is shown in Figure 8. The heart rate is calculated from the peak-peak interval of the ECG signal.

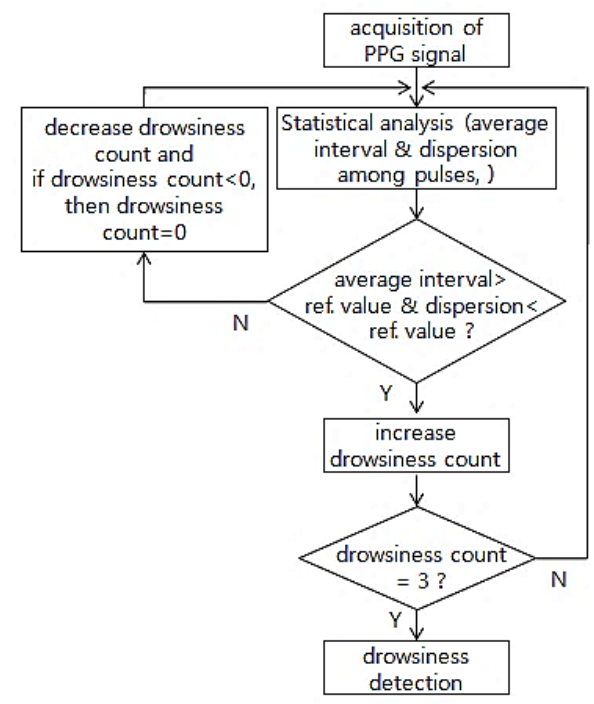

Figure 8. Drowsiness detection algorithm based on PPG signal

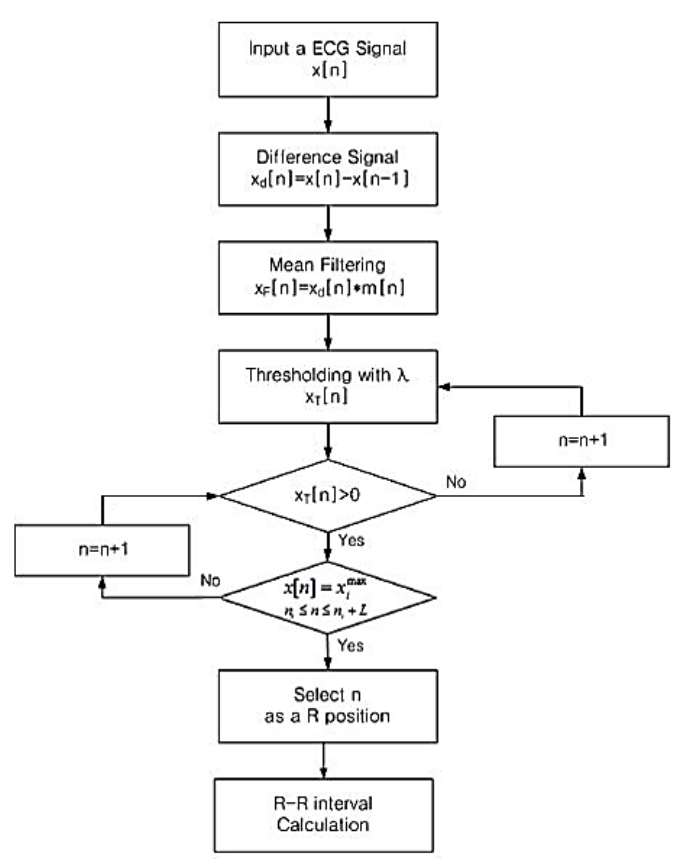

Figure 9. Heart rate detection algorithm based on ECG signal

The driver ECG signal is a reliable physiological signal for obtaining information about the heart, such as heart rate and 
heart rate variability. Finding the moments when QRS wave peak of occurs is most important for analyzing ECG signals. The interval between peaks of two neighboring QRS waves (the R-R interval) can then be easily calculated. This process is illustrated in Figure 9. Figure 10 shows how to find the $\mathrm{R}$ wave peak, start point, and end point of a QRS wave, as well as how to determine the R-R interval and $\mathrm{R}$ wave duration.

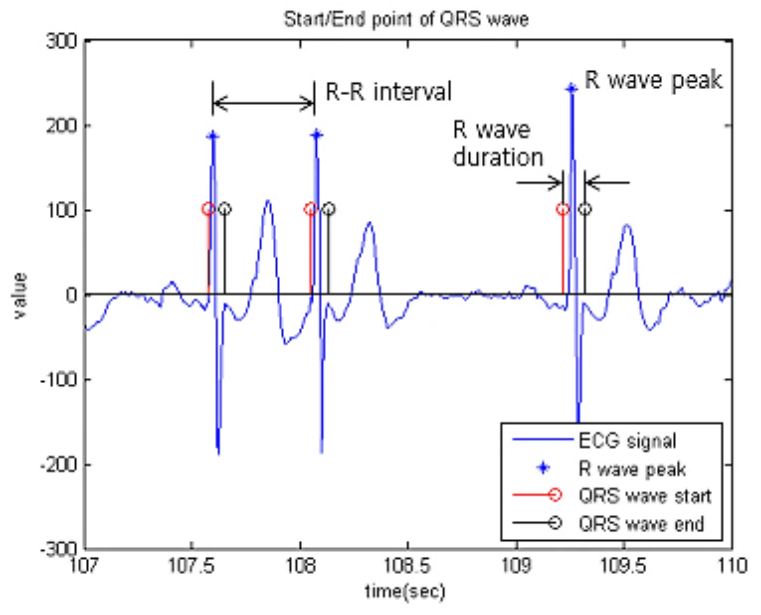

Figure 10. Determination of R-R interval and $R$ wave duration of ECG signal

Combining the above three detection algorithms, a flow chart of the entire drowsiness detection algorithm is given in Figure 11. The PPG signal and ECG signal depend on where the driver's hands are placed on the smart steering wheel, so both signals are used to ensure successful drowsiness detection. The respiration signal is always variable except when the safety belt is not fastened well. Once driver drowsiness is detected, the driver warning system is used to alert the driver.

\subsection{Arrhythmia Detection}

As one of the most common cardiac disease, driver arrhythmia can also be detected by analyzing the ECG signal. The R-R interval and $\mathrm{R}$ wave duration of the ECG signal for normal persons are in a certain range. Therefore, if they are located outside the normal range, it means that the driver is probably at risk for cardiac disease, for example arrhythmia. Figure 12 illustrates an analysis result of arrhythmia using the R-R interval and $\mathrm{R}$ wave duration of an ECG signal. The ECG signal was acquired from an arrhythmia patient in a laboratory instead of in real driving. The R-R interval and $\mathrm{R}$ wave duration defined in Figure 10 can be determined in the upper figure. There are two abnormal R-R intervals at 4,537 seconds and 4,547 seconds, which show a faster heart rate than normal persons (middle figure). The corresponding $\mathrm{R}$ wave duration at these two moments was compared with the normal value, which indicated that arrhythmia occurred two times in this period, as shown two moments with 1 value in the very bottom figure. It is necessary to use more ECG signal to validate the arrhythmia detection algorithm to ensure its reliability.

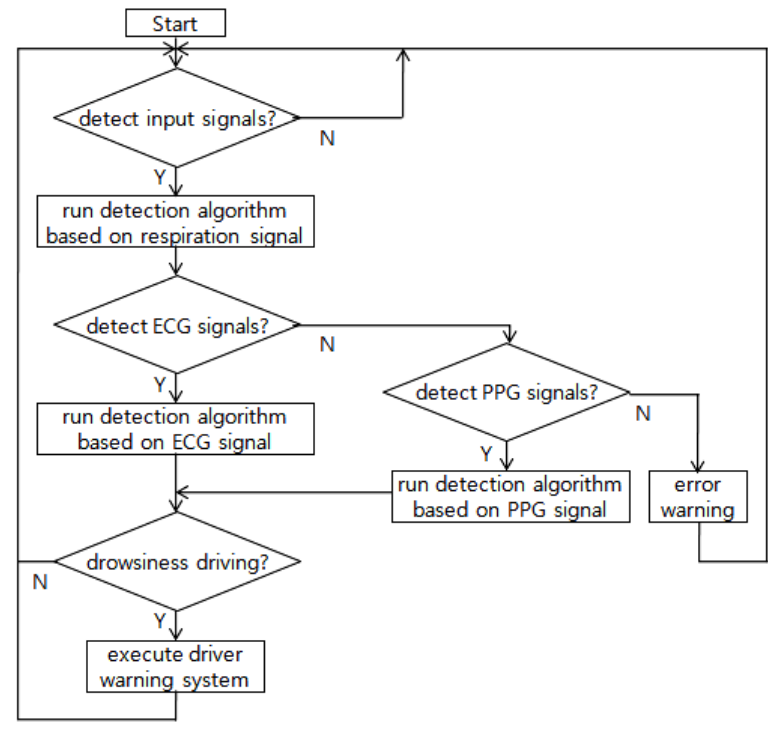

Figure 11. Flow chart of drowsiness detection algorithm
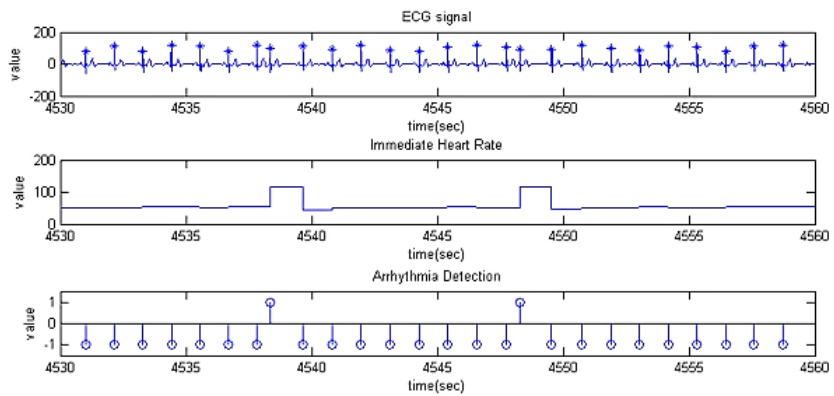

Figure 12. Detection of arrhythmia from ECG signal

\section{CONCLUSION}

Based on a smart steering wheel, a set of real-time health detection system for the driver has been proposed in this research. The proposed detection system of the smart steering wheel is composed of three biological sensors, an interface circuit, a data acquisition board, and a PC. By monitoring biological signals, the driver's health condition, including drowsiness and arrhythmia, can be detected by the developed algorithm. The test results indicate that the developed real-time driver health detection system is effective. The accuracy and reliability of the system, especially arrhythmia detection algorithm, are being validated based on more really measured data. Thus, the true positive states of drowsiness and arrhythmia can be ensured, while false positive results can be avoided as much as possible. The developed smart steering wheel could advance prognostic health management for drivers to promote intellectualization of vehicles in the near future. 


\section{ACKNOWLEDGEMENT}

The authors are grateful to Keimyung University for Professor Lee's sabbatical year to do this research from September 2016 to August 2017.

\section{REFERENCES}

Bergasa, L., Nuevo, J., Sotelo, A., Barea, R. \& Lopez, M. (2006). Real-time system for monitoring driver vigilance. IEEE Intelligent Transportation System. vol. 7, pp. 63-77

Deboleena, S., \& Madhuchhanda, M. (2012) R-peak detection algorithm for ECG using double difference and RR interval processing. Procedia Technology, vol. 4, pp. 873-877.

Eskandarian, A., \& Mortazavi, A. (2007). Evaluation of a Smart Algorithm for Commercial Vehicle Driver Drowsiness Detection. IEEE Intelligent Vehicles Symposium (553-559), June 13-15, Istanbul.

Furman, G.D., Baharav, A., Cahan, C., \& Akselrod, S. (2008). Early detection of falling asleep at the wheel: a heart rate variability appoach, Computers in Cardiology, vol. 35, pp. 1109-1120.
Gang, L., \& Chung, W. (2013). Detection of Driver Drowsiness Using Wavelet Analysis of Heart Rate Variability and a Support Vector Machine Classifier. Sensors, vol. 13, pp. 16495-16511.

Karel, A., Brookhuis, Dick, W. (2010). Monitoring drivers' mental workload in driving simulators using physiological measures. Accident Analysis and Prevention, vol. 42, pp. 898-903.

Kim, W.S., Park, S.J., Shin, J.W., \& Yo, Y.R. (2003). Analyzing heart rate variability for automatic sleep stage classification. Korean Journal of the Science of Emotion and Sensibility, vol. 6, pp. 9-14.

Lee, W.S., Jung, G.H., Hong, W.G., Park, S.W., Park, Y.S., Son, J.W., Park, S.K., \& You, H.C. (2010). Analysis of drivers' ECG biological signal under different levels of cognitive workload for intelligent vehicle. Ergonomics Society of Korea

Rosekind (2006). Underestimating the societal costs of impaired alertness: safety, health and productivity risks. Sleep Medicine. vol. 7, pp. S21-S25.

Traffic Accident Analysis Center (2012), Traffic Accident Statistical Analysis 2012, KOROAD 